\title{
Methamphetamine Use Among Patients Undergoing Methadone Maintenance Treatment in Iran; a Threat for Harm Reduction and Treatment Strategies: A Qualitative Study
}

\author{
Seyed Ramin Radfar, ${ }^{1,2,}$ Sarah J. Cousins, ${ }^{2}$ Schwann Shariatirad, ${ }^{1,3}$ Alireza Noroozi, ${ }^{4}$ and Richard A. \\ Rawson ${ }^{2}$ \\ ${ }^{1}$ Substance Abuse and Dependence Research Center, University of Social Welfare and Rehabilitation Sciences, Tehran, IR Iran \\ ${ }^{2}$ UCLA Integrated Substance Abuse Programs, Los Angeles, USA \\ ${ }^{3}$ Students Research Center of International Campus, Tehran University of Medical Sciences, Tehran, IR Iran \\ ${ }^{4}$ Iranian National Center for Addiction Studies (INCAS), Iranian Institute for Reduction of High-Risk Behaviors, School of Advanced Technologies of Medicine, Tehran \\ University of Medical Sciences, Tehran, IR Iran \\ "Corresponding author: Seyed Ramin Radfar, UCLA Integrated Substance Abuse Programs, 11075 Santa Monica Blvd., Ste 100, Los Angeles, CA, USA. Tel: +98-9131946104, E-mail: \\ raminradfar@yahoo.com
}

Received 2015 May 29; Revised 2015 July 11; Accepted 2015 July 17.

\begin{abstract}
Background: Opiates are the most commonly reported substances of abuse in Iran. Over two thirds (68\%) of all newly identified HIV cases in Iran are among people who inject drugs. On the heels of the HIV epidemic, methamphetamine use has grown. Public health officials are concerned that methamphetamine use has gained popularity among individuals who use opioid substitution therapy, such as methadone maintenance therapy.

Objectives: The purpose of this qualitative study is to inform stakeholders of the motivations and experiences of MMT patients who use methamphetamine.

Patients and Methods: To gain a better understanding of patients' motivations and experiences with MMT and methamphetamine, the authors conducted 7 focus groups with 45 participants in drop-in centers, public outpatient clinics, and a private outpatient clinic in Isfahan, Iran.

Results: Patients reported that their use of methamphetamine was motivated by methadone's side effects and encouraged by family and friends who promoted methamphetamine use to assuage the side effects of methadone in the early stages of treatment before the appearance of methadone maintenance therapy's effects on their life.

Conclusions: Findings suggest that there is a need for evidence-based practices in methadone maintenance therapy programs in Iran to reduce methamphetamine use among methadone maintenance therapy patients. Methamphetamine use among methadone maintenance therapy patients in Iran can reduce the efficacy of these services in reducing risky behaviors as well as other desired outcomes of methadone maintenance therapy. Our findings suggest two strategies that may be of use (or our findings suggest that two strategies that may be of use are: 1) educate patients and their families about methadone's side effects and the contraindications of methamphetamine use by treatment team and/or peer groups' educators; 2 ) integrate routine amphetamine testing into methadone treatment. These findings may be useful to those designing and implementing strategies for reducing methamphetamine use in methadone maintenance therapy programs in Iran.
\end{abstract}

Keywords: Methamphetamine, Iran, Harm Reduction, HIV, Qualitative

\section{Background}

HIV infection is one of the most significant social disasters worldwide and intravenous drug injection has remained as one of the routes of infection in this issue (1). In addition, HIV infection is known as a public health concern in Iran and it is estimated that $15 \%$ of all people who inject drugs (PWIDs) are HIV positive and over two thirds (68\%) of all newly identified HIV cases in Iran are among PWIDs (2). Opiates are the most commonly reported substances of abuse in Iran and the government implemented harm reduction programs such as opioid substitution therapy
(OST) to address the growing HIV epidemic among PWIDs (3-6).

Today, approximately 500,000 patients are registered for OST treatment in more than 5,000 private and public outpatient substance use disorder (SUD) treatment clinics in Iran (7).

Worldwide, methadone maintenance therapy(MMT) is one of the most common treatments used for opioid substitution therapy $(8,9)$. MMT helps to reduce injection drug use, the sharing of needles, risky sexual behaviors, and the chances of HIV infection (10-12). 
Despite the positive effects of MMT, research indicates that some methadone users may misuse other substances, such as alcohol (13-17), benzodiazepines, amphetaminetype stimulants (ATS, e.g., methamphetamine) $(18,19)$ and cocaine (20) for intoxication. Although opiates are the most frequently used substances in Iran, methamphetamine has become increasingly available and popular $(21,22)$. For instance, the proportion of reported ATS users grew from $0 \%$ in 2004 to $3.6 \%$ in 2008 (23). Moreover, in 2010 - 2011, Iran ranked among the top five countries reporting the largest ATS seizures (24-26) and also, in 2013, $80 \%$ of PWIDs reported using ATS (27).

Methamphetamine use is associated with risky sexual behaviors and injection drug use, which can increase the risk of HIV infection and other health consequences (28-30). Public health authorities in Iran are concerned about methamphetamine use among MMT patients and its threat to HIV prevention efforts $(21,31)$. Clinical observations suggest that adherent MMT patients with no prior history of ATS use have begun to use methamphetamine to cope with MMT side effects such as psychomotor retardation (31). With the exception of the study by Shariatirad et al. there is a paucity of empirical evidence of methamphetamine use among MMT patients in Iran (31).

\section{Objectives}

This study was conducted to find a reliable understanding of methamphetamine abuse among Iranian patients undergoing MMT programs in different settings qualitative methods are selected to explore topics or populations that have not been well studied (32). So, the purpose of this qualitative study is to inform stakeholders of the motivations and experiences of MMT patients who use methamphetamine.

\section{Patients and Methods}

\subsection{Design}

Focus groups discussion (FGs), is a validated technique to explore underlying norms and values (33), in this study seven FGs were formed in Isfahan, Iran, to improve understanding of patients' motivations to use methamphetamine while receiving MMT. The main objectives of the study were to obtain opinions and ideas of the patients and service providers about the roots and causes of methamphetamine abuse while the patients are under MMT and possible interventions that can be useful for prevention and/or reducing this phenomenon that potentially could decrease possible outcomes of MMT.
Seven FGs were held between December 2013 and February 2014. Participants of all focus groups were patients currently in methadone treatment. Two sessions were conducted with female participants in womenfriendly facilities and sessions with male participants included two sessions in drop-in centers, two sessions in public outpatient clinics, and one in a private outpatient clinic. The FG topics included beliefs about methamphetamine and MMT use, as well as the effects of these substances on their quality of life, health status and engagement in risky behaviors.

\subsection{Participants}

Flyers were displayed and distributed to patients in 7 clinics to invite eligible patients to one focus group (FG). Eligible participants included volunteers aged 18 and older who received methadone for at least 6 months and had started to abuse methamphetamine. Patients contacted the lead author via a telephone number written /mentioned on the flyer if they were interested in, or had questions about FG participation. The author arranged each FG based on the availability of the participants. In total, 45 participants were involved in 7 FGs. The FGs ranged in size from 5 to 7 participants, with a mean of 5 participants. Participant characteristics are displayed in Table 1.

\subsection{Data Collection}

FGs were conducted in private rooms in clinics after the working hours of clinic to reduce risk of contact with other persons. Participants were able to see and hear each other but they were identified by numbers or nick names. Also an envelope with stamps and address of PI/interviewer delivered to them and they had the opportunity even after the session to write what they were not willing to express during FG sessions but wanted to share with research team, email and phone numbers are also another options that participants could use.

A consent form (written in Persian) was read aloud to participants prior to the start of each FG session. The consent described the purpose of the study, procedures, confidentiality and the risk and benefits of participation. Upon voluntary consent, the FG facilitator provided an overview of the expectations of the FG (e.g., respect each other's opinions, allow everyone to talk in the given time, respect the confidentiality of the group). FGs were gender specific and led by same-sex facilitators. Each FG session lasted about 70 - 75 minutes and was audio-taped. The facilitator took written notes on participants' comments. The notes were then checked with the participants at the end of each session to ensure the accuracy of the notes. Participants were paid 250,000 Iranian Rials (about 9 USD). 
Table 1. Demographic Details of the Participants

\begin{tabular}{|c|c|c|c|c|c|c|c|c|}
\hline \multirow[t]{2}{*}{ Variables } & \multirow[t]{2}{*}{ FGs } & \multirow[t]{2}{*}{ Participants } & \multicolumn{4}{|c|}{ FGs From Each Center } & \multirow[t]{2}{*}{ Age, Mean } & \multirow[t]{2}{*}{ Years of Addiction, Mean } \\
\hline & & & DIC & PC & GC & WF & & \\
\hline Male & 5 & 32 & 2 & 1 & 2 & 0 & 32.4 & 9.8 \\
\hline Female & 2 & 13 & 0 & 0 & 0 & 2 & 39.3 & 7.6 \\
\hline Total & 7 & 45 & 2 & 1 & 2 & 2 & 34.4 & 9.2 \\
\hline
\end{tabular}

Abbreviations: DIC, drop in center; GC, governmental clinic; PC, private clinic; WFC, women friendly clinic.

FGs were transcribed by a study team. The PI/Interviewer listened to all of the sessions to check if any participant names were used, if so, names were erased, digitally. Recorded voices zipped and encrypted with a 6 digit password by PI/Interviewer.

All study procedures were approved by the University of California, Los Angeles, and the Tehran University of Medical Science ethics committee institutional review boards.

\subsection{Analysis}

Immediately following each FG session, the facilitator prepared a summary of the session findings. Audiotapes were transcribed verbatim by a trained transcriptionist on the study team. Transcripts were reviewed and rechecked with the recorded audios by PI and his colleagues, and edited for accuracy and completeness if needed.

After checking the transcripts with audiotapes, the transcripts were read by PI and research team (facilitator or the member that was in the FG session), in this step, firstly, the whole transcripts of each session were read and codes were extracted, secondly each question in all FGs read and again coded but not by another member of research team. Based on the objectives of the study, after that, transcripts were organized for content analysis and codes were merged or divided based on the objectives of the study using the qualitative data analysis software program ATLAS.ti (version 6).

\section{Results}

Major themes of the FGs revealed that there were several motivations and consequences of using ATS while receiving MMT. Findings did not vary by MMT setting or gender.

\subsection{Prevalence of Methamphetamine Use Among Methadone Patients}

All male participants and a majority of female ones indicated that methamphetamine use was normative among patients in MMT settings. A majority of the participants believed that those who had no history or interest in using methamphetamine at the time of admission to MMT were very likely to use methamphetamine 20 to 40 days after starting MMT. About half of the participants reported that they decided to use, or continue to use, methamphetamine during MMT.

According to the participants' opinion, there are some factors that play some role in using or not using methamphetamine during treatment with methadone. These factors categorized as:

1) Positive attitude for methamphetamine use

Participants expressed a variety of motivations to use methamphetamine while in MMT. For instance, participants reported that the effects of methadone and methamphetamine "work(ed) well together" as compared to the use of methamphetamine with other opioids such as heroin or opium. Additionally, methamphetamine was not detectable by MMT clinics that primarily test for morphine.

a) Male participant

"Everybody knows that methadone and methamphetamine work well together because both result in a negative urine test (for morphine)."

Additionally, an elevated mood and tolerance of stressful situations was reported as an effective way to cope with conflict among family members.

b) Female participant

"When I am really in conflict with my husband and kids and poverty, only methamphetamine can help me tolerate this situation."

2) Methadone's side effects

Participants were motivated to use methamphetamine in order to cope with methadone's side effects. For instance, among male participants, sexual dysfunction was a major driver of their methamphetamine use.

a) Male participant

"After a few months of MMT, I was so unable to have sex with my wife that she rebuked me as... [being] less than a rooster."

b) Male participant 
"My wife told me that I am sure you are with someone else because you are not interested...[in having] sex with me."

A majority of female participants expressed lethargy and drowsiness from methadone use as a motivator for methamphetamine use.

c) Female participant

"In the first months of treatment I was unable to do any of my household chores/housekeeping and care of my children."

3) Effects of Methamphetamine while using methadone

Almost all of the participants reported that they used methamphetamine because it reduced cravings for opioids and other drugs while they were receiving MMT. However, many believed that methamphetamine was particularly helpful in reducing the methadone dosage and treatment fatigue.

a) Female participant

"After one year of treatment, I was unable to reduce my methadone dosage and I was also really bored... [in] treatment; one of my friends told me that I can easily get rid of methadone if I use methamphetamine."

The main benefits of using methamphetamine while on MMT included increased positive mood and self-esteem. However, increased libido and energy were identified as crucial to coping with methadone's side effects, such as sexual dysfunction.

\subsection{Consequences of Methamphetamine Use}

Despite the perceived benefits of methamphetamine use, several participants experienced unwanted side effects when they increased duration and/or dosage of their methamphetamine use. Unwanted side effects included paranoia, obsessive behaviors, job loss, and the perception of diminished skills or abilities.

a) Male participant

"When I was on methadone, I was so busy with my work that I had no time even to come and take my weekly dose, but after a few months of methamphetamine use, I am not only jobless but also my wife is now living separately [from me] and we are in the process of divorce because I was very suspicious [of] her and she couldn't tolerate [it]."

b) Male Participant

"Before this, I was able to open a cell phone in 3 minutes for repair, but now I try a whole night to open it but I cannot repair it. After this failure, I use more methamphetamine to relax[ed]."

c) Female participant

"I spent a whole night...cleaning the sink and cabinets in my kitchen and I was continuing until my family sent me to [a] psychiatric hospital."
Risky sexual behaviors as a result of methamphetamine use were reported by men and women.

d) Male participant

"Prior to using methamphetamine, I used to use a condom even with my wife, but after [using methamphetamine], I had sex without a condom on many occasions with both males and females! At that time I wasn't thinking about anything, not even the risk of diseases, because I had a more enjoyable time without condom[s]."

e) Female participants

"When I started to use methadone, after a few weeks many of my clients were not satisfied with me because I was not enjoying sex when having sex with them. Then my pimp told me to use methamphetamine and the problem was solved!"

Insomnia, hepatitis, and physical side effects such as weight loss and skin problems (such as sores and dryness), were also unwelcomed. However, some participants reported that insomnia could be considered a benefit, depending on the situation.

f) Male participant

"I am a truck driver and without methamphetamine I cannot do my job [well] because it is very nice when you are always alert as a driver!"

Participants stated that they felt that patients in MMT who drop out of MMT treatment because of methamphetamine use, were more likely to become injecting drug users and/or using higher amounts of opioid drugs compared to the time before treatment.

\section{Discussion}

Phenomenology of methamphetamine abuse during MMT could be described in below steps:

1) Patient might or might not have positive attitude toward methamphetamine before starting MMT, but after beginning MMT other patients who are already under MMT may influence his/her.

2) When patients start MMT, during the induction and stabilization phases of MMT, they will frequently experience many side effects of methadone. If the treatment team fails to provide education and information during this phase, the patient may try to cope with methadone's side effects (mainly lethargy, drowsiness and sexual dysfunction) by using methamphetamine.

3) Other patients will frequently offer methamphetamine to new patients to overcome methadone's side effects. While ATS test is not done in the treatment plan of the clinics in Iran, patients can use it without being detected. If most patients who begin to use methamphetamine in the early stages of treatment won't be able 
to stabilize their lives and appreciate the full benefits of methadone treatment.

Also, we suggest two schematic diagrams for methamphetamine use and its effect on MMT programs which is shown in Figure 1.

Figure 1. Schematic Pattern for Methamphetamine use and its Effect on Methadone Maintenance Treatment

\section{A}

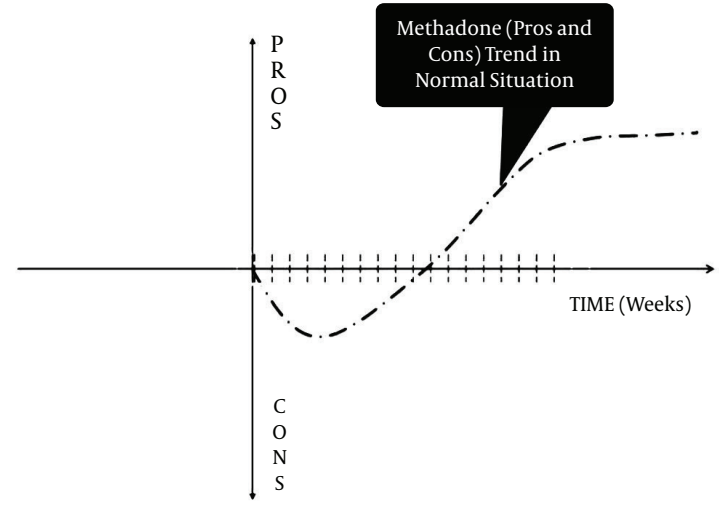

B

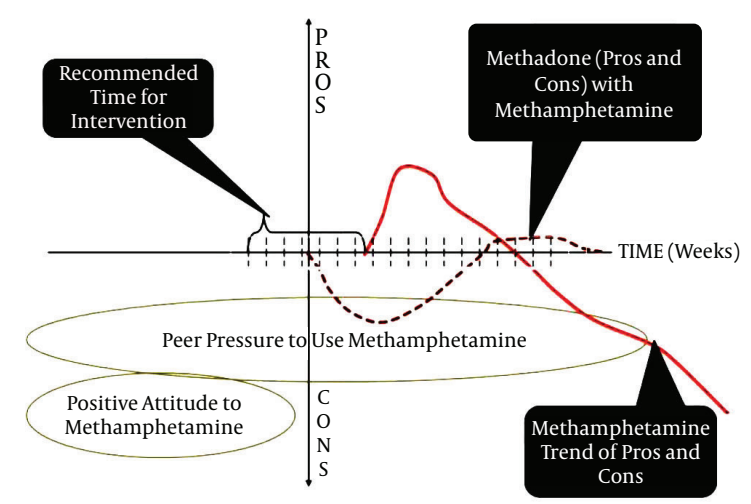

A, this diagram shows the usual process of patients undergoing MMT based on the duration, pros and cons; B, shows the process when the patients use methamphetamine and recommended time to establish interventions.

According to our findings four factors appear to promote positive attitudes about methamphetamine use for patients in MMT:1) relief from the negative side effects of methadone; 2 ) the perceived norm of methamphetamine use in OST settings; 3 ) peer pressure to use methamphetamine; and 4) lack of education about MMT side effects or methamphetamine addiction. These findings are similar to those reported by Shariatirad et al. (31).

Findings from this study warrant considerations by policy makers, public health authorities, and treatment providers. Methadone maintenance is associated with a reduction in opioid use, criminal behaviors, psychiatric symptoms, family problems, social problems, unemployment, and hepatitis and HIV infection (34). Methamphetamine use may reduce or negate these benefits. Negative side effects of methadone, such as asthenia, decreased sex drive, pain, and weight gain may contribute to poor outcomes among patients receiving MMT. Negotiating these side effects is an important clinical challenge. According to participants in our focus groups, OST clinics neither provide patients with educational information on the type of side effects MMT may cause, nor on effective and healthy ways to cope with these side effects. Improving patients' knowledge and understanding of MMT may increase treatment adherence, provide an alternative to methamphetamine use, and improve health outcomes (35).

Beliefs about the "positive" aspects of methamphetamine use, such as its "non-addictive" nature and short-term effects of use, like increased sexual libido, energy, and concentration, may influence the use of methamphetamine in light of MMT's side effects. Similar to a qualitative study in south China (36), our study found that sexual dysfunction negatively influenced the stability of maintenance treatment. To attenuate negative sexual side effects of methadone, Brown and Zueldorff suggest reducing the dose of methadone (37). Given that sexual dysfunction may be characterized by psychological, psychiatric, and neurological factors, further research is needed to address sexual dysfunction among patients receiving MMT. However, ongoing case management of adverse side effects may help to manage expected and unexpected adverse effects of MMT.

Participants in the focus groups reported that methamphetamine use was common, if not inevitable, among MMT patients. Future quantitative research should investigate the prevalence of methamphetamine use among MMT patients. If methamphetamine use is not the norm among MMT patients, it is possible that patient education can correct the misperception that it is. For instance, a social media campaign may help set a social norm, driven by a public health agenda to moderate use of methamphetamine and other drugs (38).

Employing evidence-based practices, such as establishing and maintaining abstinence from all illicit substances, developing coping, refusal, and problem-solving skills, and using motivational interviewing to initiate or maintain recovery, may be useful in improving treatment outcomes (39). Participants lacked resistance skills when offered methamphetamine from friends and family. Refusal skills have been shown to decrease tobacco and substance use (40).

Additionally, peer support educators have been shown to be successful in interventions for chronic disease man- 
agement, including substance abuse, diabetes, and HIV infection (41-44). Additionally, low-cost contingency management has been shown to increase abstinence among methamphetamine users in community-based MMT services (45).

Researchers and public health authorities should identify evidence-based interventions for MMT in Iran. If methamphetamine use occurs among MMT patients, it should be addressed as part of the initial or ongoing treatment plan. Likewise, the American Society of Addiction Medicine recommends the use of ongoing drug testing in addiction treatment settings (46). Testing for a variety of substances, including methamphetamine, may thwart methamphetamine use and improve treatment outcomes.

\subsection{Conclusions}

The rise of ATS use among MMT patients in Iran may represent a failure among OST systems (47-49) and HIV prevention efforts (50-52). Drug abuse in Iran is among top 4 diseases that cause a high burden for Iranian community both in disability adjusted life years (DALY) and years lived with disability (YLD) (53). DALYs number in Iran with 698 per100,000 population(9) is more than three times bigger than average of DALYs due to illicit drug use in the world that is 200 per 100,000 (54). This pattern is also repeated for age-standardized death rates for SUD per 100, 000 that is 4 for the global number (54) and 11.1 in Iran (9).

People who inject drugs (PWIDs) are the main population in HIV epidemic in Iran. Increasing successful rate of MMT as an approved strategy in harm reduction (55-57) not only can reduce overall burden of disease in Iran but also can reverse or stop HIV epidemic drivers. Methamphetamine use by patients on MMT can destroy many of the beneficial effects of MMT. To thwart the HIV epidemic, and decrease the drug abuse burden in Iran it is critical for policy makers and researchers to address methamphetamine use among patients receiving methadone. Future endeavors should include policy makers and government sectors in an effort to improve substance abuse treatment policies through the use of evidence-based practices. From the data in this study, two strategies that may be of use are: 1 ) educate patients and their families about methadone's side effects and the contraindications of methamphetamine use by treatment team and/or peer groups educators; 2 ) integrate routine amphetamine testing into methadone treatment.

\section{Acknowledgments}

Dr. Radfar was supported by the 2012 IAS-NIDA professional fellowship award. Dr. Rawson was supported by NIH
Fogarty grant D43 TW009102. The authors wish to thank staff at the MMT clinics and participants who took part in this study. Supports of Kris Langabeer was essential in preparing the manuscripts, authors use this opportunity to express their thanks for her supports.

\section{Footnote}

Authors' Contribution: Seyed Ramin Radfar and Richard A. Rawson contributed to the design, implementation and data analysis of the current study. Alireza Noroozi conducted and supervised focus group discussions. All authors, including Sarah J. Cousins and Schwann Shariatirad contributed to the writing of this article.

\section{References}

1. Perkins EL, Stennis KB, Taylor Spriggs V, Kwegyir-Afful EA, Prather A. Is Knowledge Enough? Considering HIV/AIDS Risk Behaviors and HIV/AIDS Knowledge with African American Women. Int J High Risk Behav Addict. 2014;3(3):ee15038. doi: 10.5812/ijhrba.15038. [PubMed: 25593891].

2. Karrari P, Mehrpour O, Afshari R, Keyler D. Pattern of illicit drug use in patients referred to addiction treatment centres in Birjand, Eastern Iran.J Pak Med Assoc. 2013;63(6):711-6. [PubMed: 23901670].

3. Eshrati B, Asl RT, Dell CA, Afshar P, Millson PM, Kamali M, et al. Preventing HIV transmission among Iranian prisoners: initial support for providing education on the benefits of harm reduction practices. Harm Reduct J. 2008;5:21. doi: 10.1186/1477-7517-5-21. [PubMed: 18541032].

4. Nissaramanesh B, Trace M, Roberts $M$. The rise of harm reduction in the Islamic Republic of Iran. Beckley Foundation Drug Policy Programme, Briefing Paper. 2005.. .

5. Razzaghi E, Nassirimanesh B, Afshar P, Ohiri K, Claeson M, Power R. HIV/AIDS harm reduction in Iran. Lancet. 2006;368(9534):434-5. doi 10.1016/S0140-6736(06)69132-0. [PubMed: 16890814].

6. Zamani S, Radfar R, Nematollahi P, Fadaie R, Meshkati M, Mortazavi $S$, et al. Prevalence of $\mathrm{HIV} / \mathrm{HCV} / \mathrm{HBV}$ infections and drugrelated risk behaviours amongst IDUs recruited through peerdriven sampling in Iran. Int J Drug Policy. 2010;21(6):493-500. doi: 10.1016/j.drugpo.2010.04.006. [PubMed: 20483578].

7. National AIDS Committee Secretariat . 2014.. Islamic Repoblic of Iran Progress Report on Monitoring of United Nations General Assembly Special Session (UNGASS) on HIV and AIDS;

8. WHO . Bulletin of the World Health Organization. ; 2008.

9. WHO. 2013..Atlas: substance use in the Eastern Mediterranean Region 2012; .

10. Chen W, Xia Y, Hong Y, Hall BJ, Ling L. Predictors of continued HIVrisk behaviors among drug users in methadone maintenance therapy program in China-a prospective study. Harm Reduct J. 2013;10:23. doi: 10.1186/1477-7517-10-23. [PubMed: 24107380].

11. Mattick RP, Breen C, Kimber J, Davoli M. Methadone maintenance therapy versus no opioid replacement therapy for opioid dependence. Cochrane Database Syst Rev. 2003(2):CD002209. doi: 10.1002/14651858.CD002209. [PubMed: 12804430].

12. Woody GE, Bruce D, Korthuis PT, Chhatre S, Poole S, Hillhouse M, et al. HIV Risk Reduction With Buprenorphine-Naloxone or Methadone: Findings From a Randomized Trial. J Acquired Immune Deficiency Syndromes. 2014;66(3):288-93. 
13. el-Bassel N, Schilling RF, Turnbull JE, Su KH. Correlates of alcohol use among methadone patients. Alcohol Clin Exp Res. 1993;17(3):6816. [PubMed: 8333601].

14. Hillebrand J, Marsden J, Finch E, Strang J. Excessive alcohol consumption and drinking expectations among clients in methadone maintenance. J Subst Abuse Treat. 2001;21(3):155-60. [PubMed: 11728789].

15. Liebson I, Bigelow G, Flamer R. Alcoholism among methadone patients: a specific treatment method. Am JPsychiatry. 1973;130(4):483-5. doi: 10.1176/ajp.130.4.483. [PubMed: 4691309].

16. Rowan-Szal GA, Chatham LR, Simpson DD. Importance of identifying cocaine and alcohol dependent methadone clients. Am J Addict. 2000;9(1):38-50. [PubMed: 10914292].

17. Raffa JD, Grebely J, Tossonian H, Wong T, Viljoen M, Khara M, et al. The impact of ongoing illicit drug use on methadone adherence in illicit drug users receiving treatment for HIV in a directly observed therapy program. Drug Alcohol Depend. 2007;89(2-3):306-9. doi:10.1016/j.drugalcdep.2007.02.007. [PubMed: 17383117].

18. Stitzer ML, Griffiths RR, McLellan AT, Grabowski J, Hawthorne JW. Diazepam use among methadone maintenance patients: patterns and dosages. Drug Alcohol Depend. 1981;8(3):189-99. [PubMed: 7327083].

19. Bryan AD, Fisher JD, Fisher WA, Murray DM. Understanding condom use among heroin addicts in methadone maintenance using the information-motivation-behavioral skills model. Subst Use Misuse. 2000;35(4):451-71. [PubMed: 10741537]

20. Johnson S, MacDonald SF, Cheverie M, Myrick C, Fischer B. Prevalence and trends of non-medical opioid and other drug use histories among federal correctional inmates in methadone maintenance treatment in Canada. Drug Alcohol Depend. 2012;124(1-2):172-6. doi: 10.1016/j.drugalcdep.2011.12.014. [PubMed: 22265193].

21. Alam Mehrjerdi Z. Crystal in Iran: methamphetamine or heroin kerack. Daru. 2013;21(1):22. doi: 10.1186/2008-2231-21-22. [PubMed 23497450].

22. Shariatirad S, Maarefvand M, Ekhtiari H. Emergence of a methamphetamine crisis in Iran. Drug Alcohol Rev. 2013;32(2):223-4. doi 10.1111/dar.12014. [PubMed: 23190186].

23. World Drug Report . 2009.. United nations office on drugs and crime;

24. UNCOD. United nations publication. ; 2007.

25. UNCOD. United Nations publication. ; 2012.

26. UNODC. United nations publication. ; 2013.

27. Radfar SR, Noroozi A, Tayeri K, Motavalian A, Nematollahi P, Mohsenifar S. Study on Prevalence of Alcohol Abuse among Injecting Drug Users in Tehran and 5 Major Cities of Iran. Asia Pac J Med Toxicol. 2014;3:20.

28. CDC . 2007.. Methamphetamine Use and Risk for HIV/AIDS; .

29. Gonzales R, Mooney L, Rawson RA. The methamphetamine problem in the United States. Annu Rev Public Health. 2010;31:385-98. doi: 10.1146/annurev.publhealth.012809.103600. [PubMed: 20070191].

30. Alam Mehrjerdi Z, Abarashi Z, Mansoori S, Deylamizadeh A, Salehi Fadardi J, Noroozi A, et al. Methamphetamine use among Iranian heroin kerack-dependent women: implications for treatment. Int J High Risk Behav Addict. 2013;2(1):15-21. doi: 10.5812/ijhrba.10216. [PubMed: 24971266].

31. Shariatirad S, Maarefvand M, Ekhtiari H. Methamphetamine use and methadone maintenance treatment: an emerging problem in the drug addiction treatment network in Iran. Int J Drug Policy. 2013;24(6):e115-6. doi: 10.1016/j.drugpo.2013.05.003. [PubMed 23773684].

32. Bernard HR. Research methods in anthropology: Qualitative and quantitative approaches. Rowman Altamira; 2011.

33. Shedlin MG, Schreiber JM. Using focus groups in drug abuse and HIV/AIDS research. NIDA Res Monogr. 1995;157:136-55. [PubMed: 8684435].

34. Center for Substance Abuse Treatment . Medication-assisted treatment for opioid addiction in opioid treatment programs. 2005.

35. Alves P, Winstock A. Patients' knowledge about treatment for opiate dependence. Psychiatrist. 2011;35(12):448-53. doi: 10.1192/pb.bp.111.034546.

36. Xia Y, Zhang D, Li X, Chen W, He Q, Jahn HJ, et al. Sexual dysfunction during methadone maintenance treatment and its influence on patient's life and treatment: a qualitative study in South China. Psychol Health Med. 2013;18(3):321-9. doi: 10.1080/13548506.2012.729845. [PubMed: 23092392].

37. Brown RT, Zueldorff M. Opioid substitution with methadone and buprenorphine: sexual dysfunction as a side effect of therapy. Heroin Addict Relat Clin Probl. 2007;9(1):35-44.

38. Bewick BM, Bell D, Crosby S, Edlin B, Keenan S, Marshall K, et al. Promoting improvements in public health: Using a Social Norms Approach to reduce use of alcohol, tobacco and other drugs. Drugs: Educ Prevent Policy. 2013;20(4):322-30.

39. McGovern MP, Carroll KM. Evidence-based practices for substance use disorders. Psychiatr Clin North Am. 2003;26(4):991-1010. doi: 10.1016/s0193-953x(03)00073-x.

40. Sussman S. A Lifespan Developmental-Stage Approach to Tobacco and Other Drug Abuse Prevention. ISRN Addict. 2013;2013:745783. doi: 10.1155/2013/745783. [PubMed: 25298961].

41. Parent N, Fortin F. A randomized, controlled trial of vicarious experience through peer support for male first-time cardiac surgery patients: impact on anxiety, self-efficacy expectation, and self-reported activity. Heart Lung. 2000;29(6):389-400. doi: 10.1067/mhl.2000.110626. [PubMed: 11080319].

42. Pearson CR, Micek MA, Simoni JM, Hoff PD, Matediana E, Martin DP, et al. Randomized control trial of peer-delivered, modified directly observed therapy for HAART in Mozambique. I Acquir Immune Defic Syndr. 2007;46(2):238-44. doi: 10.1097/QAI.0b013e318153f7ba. [PubMed: 17693890].

43. Taleghani F, Babazadeh S, Mosavi S, Tavazohi H. The effects of peer support group on promoting quality of life in patients with breast cancer. Iran J Nurs Midwifery Res. 2012;17(2 Suppl 1):S125-30. [PubMed: 23833594].

44. Wilson W, Pratt C. The impact of diabetes education and peer support upon weight and glycemic control of elderly persons with noninsulin dependent diabetes mellitus (NIDDM). Am J Public Health. 1987;77(5):634-5. [PubMed:3565666].

45. Peirce JM, Petry NM, Stitzer ML, Blaine J, Kellogg S, Satterfield $\mathrm{F}$, et al. Effects of lower-cost incentives on stimulant abstinence in methadone maintenance treatment: a National Drug Abuse Treatment Clinical Trials Network study. Arch Gen Psychiatry. 2006;63(2):201-8. doi: 10.1001/archpsyc.63.2.201. [PubMed:16461864].

46. American Society of Addiction Medicine (ASAM). 2013.. Drug Testing: A White Paper of the American Society of Addiction Medicine (ASAM);

47. Booth RE, Corsi KF, Mikulich-Gilbertson SK. Factors associated with methadone maintenance treatment retention among streetrecruited injection drug users. Drug Alcohol Depend. 2004;74(2):17785. doi:10.1016/j.drugalcdep.2003.12.009. [PubMed: 15099661]

48. DeMaria PJ, Sterling R, Weinstein SP. The effect of stimulant and sedative use on treatment outcome of patients admitted to methadone maintenance treatment. Am J Addict. 2000;9(2):145-53. [PubMed: 10934576].

49. Torrens M, Castillo C, Perez-Sola V. Retention in a low-threshold methadone maintenance program. Drug Alcohol Depend. 1996;41(1):55-9. [PubMed: 8793310].

50. Frosch D, Shoptaw S, Huber A, Rawson RA, Ling W. Sexual HIV risk among gay and bisexual male methamphetamine abusers. J Substance Abuse Treat. 1996;13(6):483-6. doi: 10.1016/s07405472(96)00098-0.

51. Halkitis PN, Parsons JT, Stirratt MJ. A double epidemic: crystal methamphetamine drug use in relation to HIV transmission among gay men. J Homosex. 2001;41(2):17-35. doi: 10.1300/J082v41n02_02. [PubMed: 11482426] 
52. Molitor F, Truax SR, Ruiz JD, Sun RK. Association of methamphetamine use during sex with risky sexual behaviors and HIV infection among non-injection drug users. West J Med. 1998;168(2):93-7. [PubMed: 9499742].

53. Naghavi M, Abolhassani F, Pourmalek F, Lakeh M, Jafari N, Vaseghi S, et al. The burden of disease and injury in Iran 2003. Popul Health Metr. 2009;7:9. doi:10.1186/1478-7954-7-9. [PubMed: 19527516].

54. WHO .2010.. Atlas on substance use (2010): resources for the prevention and treatment of substance use disorders; .

55. Gibson DR, Flynn NM, McCarthy JJ. Effectiveness of methadone treat- ment in reducing HIV risk behavior and HIV seroconversion among injecting drug users. AIDS. 1999;13(14):1807-18. [PubMed: 10513638].

56. Brugal MT, Domingo-Salvany A, Puig R, Barrio G, Garcia de Olalla P, de la Fuente L. Evaluating the impact of methadone maintenance programmes on mortality due to overdose and aids in a cohort of heroin users in Spain. Addiction. 2005;100(7):981-9. doi: 10.1111/j.1360 0443.2005.01089.x. [PubMed: 15955014].

57. Rhodes T, Hedrich D. Office for Official Publications of the European Communities. ; 2010. 